\title{
Propiedades psicométricas de una escala para medir desconexión moral en niños mexicanos
}

\author{
FERNANDA INÉZ GARCÍA VÁZQUEZ* \\ Instituto Tecnológico de Sonora, México \\ ORCID: https://orcid.org/0000-0002-8668-2924 \\ ÁNGEL ALBERTO VALDÉS CUERVO \\ Instituto Tecnológico de Sonora, México \\ ORCID: https://orcid.org/0000-0001-6559-4151 \\ ERNESTO ALONSO CARLOS MARTÍNEZ \\ Instituto Tecnológico Superior de Cajeme, México \\ ORCID: https://orcid.org/0000-0002-5432-0443 \\ CAROLINA ALCÁNTAR NIEBLAS \\ Instituto Tecnológico de Sonora, México \\ ORCID: https://orcid.org/0000-0002-1125-6437
}

\begin{abstract}
How to quote this article: García Vásquez, F.I., Valdés Cuervo, A.A., Carlos Martínez, E.A. \& Alcántar Nieblas, C. (2019). Propiedades Psicométricas de una Escala para Medir Desconexión Moral en Niños Mexicanos. Acta Colombiana de Psicología, 22(1), 107-117. doi: http://www. dx.doi.org/10.14718/ACP.2019.22.1.6
\end{abstract}

Resumen

La desconexión moral tiene como consecuencia efectos negativos para el desarrollo psicosocial en la niñez, por lo cual se hace necesario contar con escalas para evaluarla. En el presente estudio se analizaron las propiedades psicométricas de la escala Desconexión Moral en Situaciones de Acoso en niños (DMAE). Participaron 661 estudiantes mexicanos de 5. ${ }^{\circ}$ $(51 \%)$ y $6 .^{\circ}$ grado $(49 \%), 48 \%$ niñas $(M$ edad $=10.51, D E=.64$ años $)$ y $52 \%$ niños $(M$ edad $=10.59, D E=.68$ años $)$. Se analizó la validez (estructura interna y concurrente), invarianza de medida para ambos sexos y fiabilidad de la escala. De los resultados del análisis factorial confirmatorio se infirió que el modelo de medición multidimensional que mide la justificación moral, la difusión de la responsabilidad y la atribución de la culpa presenta mejor ajuste a los datos que el unidimensional. Además, se encontró que la DMAE presenta invariancia de medición en ambos sexos y evidencias de validez concurrente. Se concluye que la escala cuenta con propiedades psicométricas adecuadas para la medición de la desconexión moral en niños mexicanos.

Palabras claves: medición, validez, desconexión moral, emociones morales, acoso escolar.

\section{Psychometric Properties of a Scale Measuring Moral Disengagement in Mexican Children}

\begin{abstract}
Moral disengagement has negative effects for psychosocial development in childhood, which makes it necessary to have scales to evaluate it. The present study aimed to analyze the psychometric properties of the Moral Disengagement Scale for Children in Bullying Situations (DMAE, for its Spanish acronym) scale. 661 Mexican students participated from 5th grade $(51 \%)$ and 6 th grade $(49 \%), 48 \%$ girls $(M$ age $=10.51, S D=.64$ years) and 52\% boys ( $M$ age $=10.59, S D=.68$ years). The validity (internal and concurrent), measurement invariance for both sexes and reliability of the scale were analyzed. From the results of the confirmatory factor analysis, it was inferred that the multidimensional measurement model that measures moral justification, diffusion of responsibility and attribution of blame presents a better fit to the data than the one-dimensional one. It was also found that the DMAE presents measurement invariance in both sexes and evidence of concurrent validity. It was concluded that the scale has adequate psychometric properties for the measurement of moral disengagement in Mexican children

Keywords: measurement, validity, moral disengagement, moral emotions, bullying.
\end{abstract}

\footnotetext{
Departamento de Educación, Calle 5 de febrero 818 sur, Colonia Centro, Ciudad Obregón, Sonora, México, 0526444100900, fernanda. garcia@itson.edu.mx
}

Recibido, marzo 28/2018; Concepto de evaluación, mayo 4/2018; Aceptado, mayo 22/2018 


\title{
Propriedades psicométricas de uma escala para medir desconexáo moral em crianças mexicanas
}

\begin{abstract}
Resumo
A desconexão moral tem como consequência efeitos negativos para o desenvolvimento psicossocial na infância, o que torna necessário contar com escalas para avaliá-la. Neste estudo, foram analisadas as propriedades psicométricas da escala Desconexão Moral em Situações de Assédio em Crianças. Participaram 661 estudantes mexicanos do $5^{\circ}(51 \%)$ e $6^{\circ}$ anos $(49 \%), 48 \%$ meninas $(\mathrm{M}$ idade $=10.51, \mathrm{DP}=.64$ anos $)$ e $52 \%$ meninos $(\mathrm{M}$ idade $=10.59, \mathrm{DP}=.68$ anos $)$. Foi analisada a validade (estrutura interna e concorrente), invariância de medida para ambos os sexos e confiabilidade da escala. Dos resultados da análise fatorial confirmatória, foi inferido que o modelo de medição multidimensional que mede a justificativa moral, a difusão da responsabilidade e a atribuição da culpa apresenta melhor ajuste aos dados do que o unidimensional. Além disso, foi constatado que a escala apresenta invariância de medida em ambos os sexos e evidências de validade concorrente. Concluise que a escala possui propriedades psicométricas adequadas para a medição da desconexão moral em crianças mexicanas. Palavras-chaves: medição, validade, desconexão moral, emoções morais, assédio escolar.
\end{abstract}

\section{Introducción}

La violencia escolar se relaciona con la presencia de dificultades en el desarrollo psicosocial de los estudiantes que participan en ella. En las víctimas, por ejemplo, se reportan dificultades académicas, depresión y problemas de adaptación social (Arseneault et al., 2006; Espegale, Hong, Rao \& Low, 2013), mientras que en los agresores se describen problemas de disciplina en la escuela, abuso de sustancias y conductas disociales (Feldman et al., 2014; Kim, Catalano, Haggerty \& Abbott, 2011). Este problema, que involucra agresiones repetidas e intencionales hacia pares que no pueden defenderse por sí solos (Olweus, 1991; Volk, Dane \& Marini, 2014), se presenta con frecuencia en distintas regiones del mundo (Craig et al., 2009; Eljach, 2011; Garaigordobil \& Machimbarrena, 2017; Higuita-Gutiérrez $\&$ Cardona-Arias, 2017), y en México, en particular, afecta a un número considerable de estudiantes (Instituto Nacional de Estadística y Geografía [INEGI], 2015; Mendoza, Rojas \& Barrera, 2017; Valdés, Martínez \& Carlos, 2018).

El estudio de la moralidad ha demostrado ser un campo fructífero para la comprensión del acoso entre estudiantes (Thornberg, Pozzoli, Gini \& Jungert, 2015; Valdés, Carlos, Wendlandt \& Ramírez, 2016). La conducta moral se asocia tanto a los juicios morales -que involucran valoraciones de los efectos de las conductas o situaciones sociales para el bienestar de los otros (Malti \& Ongley, 2014; Paxton \& Greene, 2010)- como a las emociones morales -es decir, experiencias afectivas asociadas a situaciones sociales que implican normas morales (Tangney, Stuewig \& Mashek, 2007)- que se encuentran involucradas en las situaciones de violencia escolar (Nucci, 2001; Smetana, 2013).
La teoría cognitiva-social sostiene que el dominio moral se relaciona con preocupaciones del individuo acerca de los efectos de su conducta en el bienestar de los otros (Gibbs, 2014; Smetana, Jambon \& Ball, 2014). El dominio moral juega un papel central en la regulación del comportamiento social, sobre todo porque sus normas actúan en diferentes contextos sin necesidad de figuras de autoridad, recompensas, castigos o presión social (Levasseur, Desbiens \& Bowen, 2017; Smetana, 2006).

Durante la niñez se desarrollan estándares morales que permiten distinguir lo correcto de lo incorrecto moralmente dentro de un contexto cultural (Echavarría \& Vasco, 2006; Hymel \& Bonnano, 2014), por lo que el cumplimiento de las normas morales es motivo de orgullo para el individuo, mientras que su violación se vincula con emociones de vergüenza y culpa (Bandura, 1999). Sin embargo, en ocasiones existe contradicción entre los estándares morales y la conducta de las personas (Caravita, Sijtsema, Rambaran \& Gini, 2014). Para explicar esta situación, Bandura (2002) utiliza el constructo de desconexión moral, el cual abarca mecanismos cognitivos que disminuyen el malestar ocasionado por las conductas que transgreden los estándares personales o las normas morales (Bandura, Barbaranelli, Caprara \& Pastorelli, 1996).

Según Paciello, Fida, Tramontano, Lupinetti y Caprara (2008), estos mecanismos se enfocan en la conducta, ya que provocan la valoración positiva del comportamiento no moral (con la justificación moral, la comparación ventajosa y las etiquetas eufemísticas, por ejemplo), la disminución de la responsabilidad (con el desplazamiento y la difusión de la responsabilidad, por ejemplo), la distorsión de las consecuencias de la conducta para la víctima (por ejemplo, 
al ignorar, minimizar o distorsionar los daños) y la desvalorización de la víctima (por ejemplo, al deshumanizarla o atribuirle la culpa).

Adicionalmente, la evidencia sugiere que mediante los mecanismos de desconexión moral se justifica (Aly, Taylor \& Karnovsky, 2014; Bandura, 1986) y se desvirtúan las causas, las consecuencias y la responsabilidad por la conducta no moral hacia los otros (Almeida, Correia \& Marinho, 2010; Bandura, 1990). La desconexión moral se vincula a conductas disruptivas, tales como el uso de sustancias (Newton, Havard \& Teesson, 2012; Passini, 2012), el comportamiento delictivo (Newton \& Bussey, 2012) y el acoso hacia los pares (Gini, Pozzoli \& Hymel, 2014; Graham, 2016; Valdebenito, Ttofi \& Eisner, 2015).

La desconexión moral es un constructo valioso en la investigación del acoso escolar (Campaert, Nocentini \& Menesini, 2017; Romero \& Kyriacou, 2016; Thornberg \& Jungert, 2014; Van Noorden, Haselager, Cillessen \& Bukowski, 2014), puesto que se ha constatado que se vincula con mayor frecuencia a la agresión hacia los pares (Menesini, Palladino \& Nocentini, 2015; Obermann, 2011; Wang, Ryoo, Swearer, Turner \& Goldberg, 2017) y al rol de espectador alentador ante el acoso (Levasseur et al., 2017; Wang, Yang, Gao, Lei \& Wang, 2017).

Sin embargo, si bien hay varios estudios enfocados en la validación de escalas de medición de la desconexión moral (Çapan \& Bakioglu, 2016; Gini, Pozzoli \& Bussey, 2015; Rubio-Garay, Amor \& Carrasco, 2017) aún persisten controversias respecto a la medición de este constructo (Greenhalgh, Watt \& Schutte, 2015), sobre todo relacionadas con: (a) la estructura interna de las escalas, porque aunque la escala original mide ocho mecanismos de desconexión moral (Bandura et al., 1996), se han reportado modelos unidimensionales (Çapan \& Bakioglu, 2016; Pelton, Gound, Forehand \& Brody, 2004), o de tres (Rubio-Garay et al., 2017) y cuatro factores (Newton, Stapinski, Champion, Teesson \& Bussey, 2016); (b) la medición de la desconexión moral, debido a que se evalúa tanto de forma general como de forma contextual a una situación; y (c) las técnicas de medición, ya que se ha evaluado el constructo mediante dilemas éticos (Paulino Avilés \& Fonseca, 2016; Stevens, Deuling \& Armenakis, 2012), entrevistas (De Graaff, Schut, Verweij, Vermetten \& Giebels, 2016) y escalas de auto-reporte tipo Likert (Çapan \& Bakioglu, 2016; Newton et al., 2016; Paciello et al., 2008).

Además, de la revisión de la literatura se infieren algunas limitaciones con respecto a la medición de la desconexión moral, ya que, por ejemplo: (a) se identificó una sola escala que mide el constructo en niños (suecos) de $10 \mathrm{a}$ 14 años en el contexto específico del acoso escolar-que, específicamente, mide siete mecanismos: justificación moral, lenguaje eufemístico, comparaciones ventajosas, desplazamiento de la responsabilidad, difusión de la responsabilidad, distorsión de las consecuencias y atribución a la víctima- (Thornberg \& Jungert, 2014), no obstante, la evidencia disponible sugiere que los mecanismos de desconexión moral son influidos por el contexto social de la conducta (Bandura, 1999; Boardley \& Kavusannu, 2007; Greenhalgh et al., 2015; Houwing \& Bussey, 2017; Moore, 2015), y que la expresión de los mecanismos de desconexión moral en la niñez y adolescencia está influida por sus padres, sus pares y las normas de su salón de clases (Camodeca \& Taraschi, 2015; Caravita et al., 2014; Pozzoli, Gini \& Vieno, 2012); (b) no se localizaron estudios que comparen el ajuste de diferentes modelos teóricos de medición del constructo en situaciones de acoso escolar; sin embargo, como se mencionó antes, existen diferencias en lo relativo a la estructura interna de las escalas de medición descritas en la literatura; (c) no se hallaron estudios que analicen la invarianza de medida en ambos sexos de las escalas a pesar de que hay diferencias reportadas en la frecuencia con que chicos y chicas utilizan mecanismos de desconexión moral (Almeida et al., 2010; Kokkinos, Voulgaridou, Mandrali \& Parousidou, 2016; Menesini et al., 2015), por lo que es necesario precisar si estas diferencias se deben a un sesgo de medición o si implican distintos niveles de expresión del rasgo; y (d) no se identificaron estudios desarrollados en México que reporten propiedades psicométricas robustas de escalas para medir la desconexión moral en niños en situaciones de acoso.

En este contexto, el presente estudio se propone desarrollar una escala con propiedades psicométricas robustas para medir la Desconexión Moral en Situaciones de Acoso Escolar en Niños Mexicanos (DMAE).

La selección de los mecanismos de desconexión moral medidos en la DMAE responde a consideraciones teóricas y prácticas. Desde el ángulo teórico, se cuida la consistencia de la escala con la propuesta de Bandura (1990, 1999, 2002), es decir, se miden los mecanismos enfocados en la conducta (justificación moral), la responsabilidad personal (difusión de la responsabilidad) y en quien recibe la agresión (atribución de culpa). Y, desde el punto de vista práctico, se incluyen mecanismos identificados en los grupos de enfoque realizados con estudiantes mexicanos, lo cual favorece la sensibilidad cultural de la escala, a la vez que se valora que en la literatura se vinculan los mecanismos medidos con baja sensibilidad moral y conductas de acoso (Perren, Gutzwiller-Helfenfinger, Malti \& Hymel, 2012; Malti, Gasser \& Buchmann, 2009; Pozzoli et al., 2012; Robson \& Witenberg, 2013; Thornberg \& Jungert, 2014).

Como resultado de estos dos tipos de consideraciones, en la DMEA se miden los mecanismos de: (a) la justificación 
moral, que implica evaluar un comportamiento dañino como útil y socialmente digno (De Caroli \& Sagone, 2014; Thornberg \& Jungert, 2014); (b) la difusión de la responsabilidad, que involucra diluir la culpa por la conducta no moral en el grupo (Bandura, Underwood \& Fromson, 1975; Caravita et al., 2014) y (c) la atribución de culpa, donde se considera que la conducta agresiva es provocada por la víctima (Newton et al., 2016; Pozzoli et al., 2012).

Finalmente, en este estudio se analiza la validez de contenido de la escala mediante el juicio de expertos, y, posteriormente, se realiza una comparación de un modelo de tres dimensiones con un modelo alternativo unidimensional (véase Figura 1) para indagar en la validez de la estructura interna y se evalúa la invarianza de medida para ambos sexos de la escala. Y, por último, se analizan las evidencias de validez concurrente mediante la relación de los puntajes de la escala con la empatía y compasión; esto debido a que la evidencia disponible sugiere que la desconexión moral se relaciona de forma negativa con ambas emociones morales (Barriga, Sullivan-Cosetti \& Gibbs, 2009; Thornberg et al., 2015).

\section{Método}

\section{Participantes}

Se eligieron de forma no probabilística 18 escuelas primarias públicas urbanas de tres ciudades (seis por ciudad) del estado de Sonora, México. El criterio inicial de inclusión fue la disponibilidad de los directivos de las escuelas para participar en el estudio. Posteriormente, mediante un muestreo probabilístico simple con reemplazo $(p=.5$, $q=95 \%$, error $=5 \%$ ), se seleccionó a 661 estudiantes de los grados $5 .^{\circ}(51 \%)$ y $6 .^{\circ}, 49 \%$ niñas $(M$ edad $=10.51$, $D E=.64$ años $)$ y $51 \%$ niños $(M$ edad $=10.59, D E=.68$ años) con un rango de edad entre los 9 y los 13 años.

\section{Instrumentos}

\section{Desconexión moral.}

Con base en la revisión de la literatura (Bandura et al., 1996; Thornberg \& Jungert, 2014), se desarrolló una escala para medir la Desconexión Moral en Situaciones de Acoso en Niños (DMAE), que está compuesta por 11 ítems que miden los mecanismos de: (a) Justificación moral (cuatro ítems, p. ej.: "Los agresores con su conducta evitan que los agredan a ellos"), (b) Difusión de la responsabilidad (tres ítems, p. ej.: "Casi todos los estudiantes se pegan entre sí") y (c) Atribución de la culpa (cuatro ítems, p. ej.: "Las víctimas tienen la culpa que los agredan por no defenderse"). Se utilizó un formato de respuesta tipo Likert que va de 0 (nunca), 1 (casi nunca), 2 (a veces) y 3 (casi siempre), a 4 (siempre).

\section{Empatía-compasión.}

Se empleó la escala "The Adolescent Measure of Empathy \& Sympathy" (AMES, Vossen, Piotrowski \& Valkenburg, 2015) que mide la empatía cognitiva (tres ítems, $\Omega=72$ ), es decir, la comprensión del estado emocional del otro (p. ej.: "Puedo entender cómo otro compañero se siente cuando es
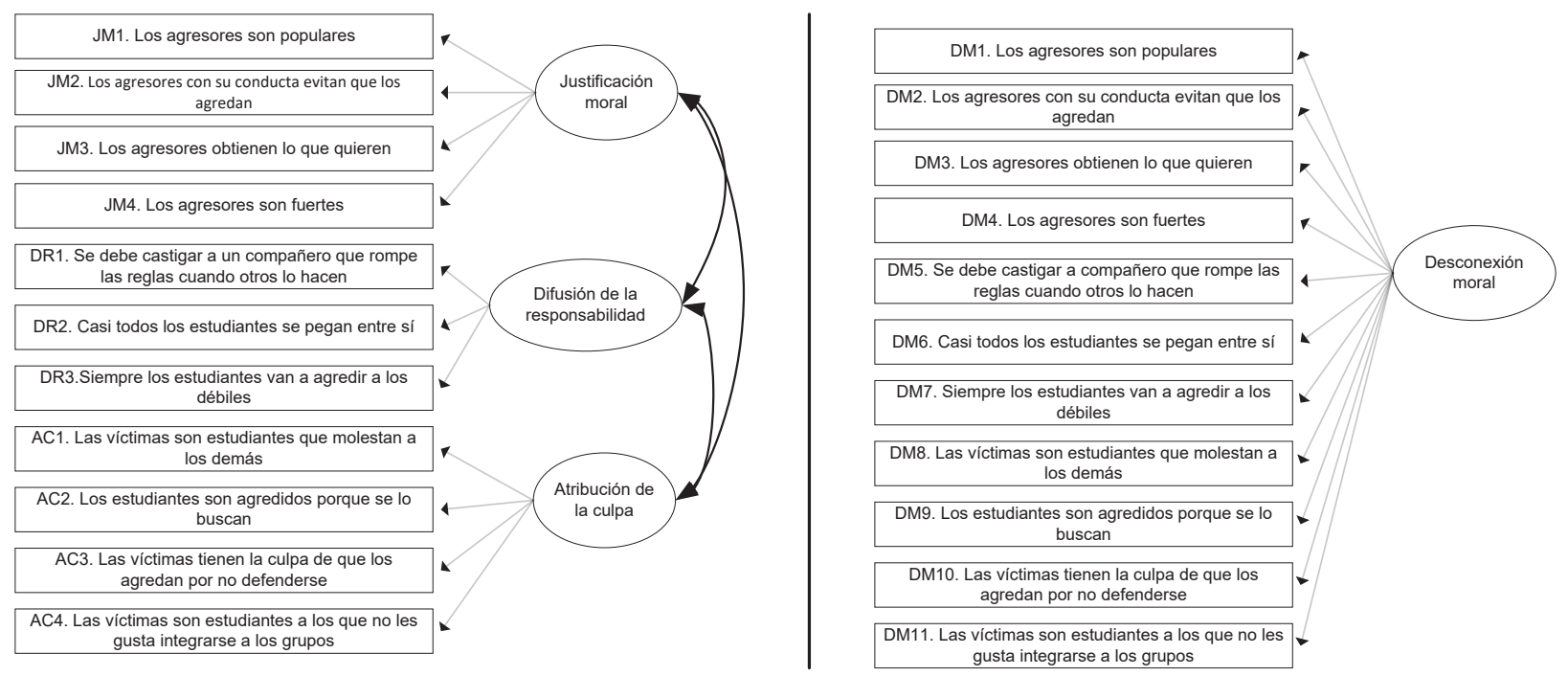

Figura 1. Propuestas teóricas para medir los roles de estudiantes espectadores de acoso. 
agredido incluso antes que me diga"); la empatía afectiva (tres ítems, $\Omega=74$ ), es decir, experimentar las emociones de otras personas (p. ej.: "Cuando un compañero está triste me siento triste también"); y la compasión (tres ítems, $\Omega=$ 71), es decir, sentir preocupación o tristeza por la situación de otra persona (p. ej.: "Me siento preocupado cuando un compañero se siente mal"). Las respuestas se presentaban en formato tipo Likert con opciones desde 0 (nunca) hasta 4 (siempre), y, para formar el índice denominado "empatíacompasión", se sumaron los valores de las tres dimensiones. Adicionalmente, mediante un análisis factorial confirmatorio se constató el ajuste del modelo de medición a los datos $\left(X^{2}=24.32, g l=17, p=.111 ; \mathrm{SRMR}=.05 ; \mathrm{AGFI}=.97\right.$; $\mathrm{CFI}=.99 ; \mathrm{RMSEA}=.03$ IC $90[.01-.06])$.

\section{Procedimiento}

Se realizaron tres grupos de enfoque, cada uno con 12 estudiantes de primaria. Inicialmente, se le presentó a los participantes situaciones de agresión entre pares, se les solicitó que elaboraran razones para explicar la conducta de los agresores, y sus respuestas se clasificaron en los mecanismos de justificación moral, difusión de la responsabilidad o atribución de la culpa. Posteriormente, se les presentó la definición de dichos mecanismos y se les pidió que describieran comportamientos que ejemplificaran su utilización en situaciones de acoso entre estudiantes.

Con base en el análisis de la información recabada en los grupos de enfoque, se elaboró una primera versión del instrumento que constó de 15 ítems (cinco por cada mecanismo). Esta primera versión sometió a pruebas de validez de contenido (teórico, cultural y lingüístico) por medio de un juicio de expertos (tres investigadores en la temática y cinco docentes), tras lo cual se consideró mantener únicamente los 11 ítems que obtuvieron un índice Kappa de concordancia entre jueces $\geq .80$.

Finalmente, para obtener la información que se analiza psicométricamente, se presentó el objetivo del estudio a los directivos de las escuelas seleccionadas y se solicitó su autorización para acceder a los estudiantes. Después de esto, se procuró por el consentimiento escrito de los padres para que los hijos contestaran la escala, y dos investigadores participantes en el proyecto realizaron la administración de los cuestionarios en los salones de clases habituales de los estudiantes. La duración de la aplicación fue, en promedio, de 20 minutos.

\section{Análisis de datos}

En primer lugar, se calcularon las medias, desviaciones estándar, asimetría y curtosis de los ítems, y, en segundo lugar, se calculó un análisis factorial confirmatorio (AFC) con apoyo del AMOS v. 22. El cálculo del AFC se llevó a cabo por medio del método de estimación de máxima verosimilitud (ML), en donde se utilizó un bootstrap con 500 repeticiones -con un intervalo de confianza del $95 \%$-para procurar que los cálculos no se afectaran por problemas de normalidad multivariada. Los valores perdidos se trataron mediante el método de imputación por regresión.

Se utilizaron los índices de ajuste $X^{2}$ (Chi-cuadrada), SRMR (raíz cuadrada de residual estandarizada), AGFI (índice de bondad de ajuste ajustado), CFI (índice de ajuste comparativo) y RMSEA IC 90 (error de la raíz cuadrada de la media de aproximación con su intervalo de confianza), donde se consideraron como aceptables los valores de $X^{2}$ con $p>.001 ;$ CFI y AGFI $\geq .95 ;$ y SRMR y RMSEA $\leq .05$ (Blunch, 2013; Byrne, 2010). Para comparar el ajuste de los modelos, se emplearon el AIC (criterio de información de Akaike) y el BIC (criterio de información Bayesiano). En ambos casos se considera que valores menores indican mejor ajuste (Kline, 2016; Wang \& Wang, 2012).

Para el análisis de la invarianza de medida de la escala en ambos sexos se estimaron progresivamente modelos anidados, los cuales se compararon entre sí. En el primer modelo no se establecieron restricciones (invarianza configural); en el segundo se incluyeron restricciones referidas a los coeficientes de regresión (invarianza métrica); en el tercero se agregaron restricciones a las cargas factoriales y a los interceptos de los factores (invarianza escalar); y, finalmente, en el cuarto modelo se impusieron restricciones en lo relativo a equivalencias entre cargas factoriales, interceptos, covarianzas y varianzas residuales (invarianza residual). Se utilizaron como indicadores de invarianza diferencias $\Delta X^{2} \operatorname{con} p>.001$, y $\Delta$ CFI menores a .01 (Blunch, 2013; Byrne, 2010).

La validez de concurrente de la escala se indagó mediante un modelo de regresión múltiple, con apoyo del software AMOS .23. Finalmente, se analizaron los efectos de los mecanismos de desconexión moral (justificación moral, difusión de la responsabilidad y atribución de la culpa) en las emociones de empatía-compasión.

\section{Resultados}

\section{Análisis descriptivos}

En la Tabla 1 se muestra que los estudiantes utilizan con escasa frecuencia los mecanismos de desconexión moral. Los valores de asimetría y curtosis sugieren la existencia de normalidad univariada en la distribución de los puntajes de los ítems. 
112

Tabla 1.

Medias, desviaciones estándar, asimetría y curtosis de los items de DMAE

\begin{tabular}{ccccccccc}
\hline \multirow{2}{*}{ Ítems } & \multicolumn{3}{c}{$M$} & \multicolumn{2}{c}{$D E$} & \multicolumn{2}{c}{ Asimetría } & \multicolumn{2}{c}{ Curtosis } \\
\cline { 2 - 9 } & $\mathrm{M}$ & $\mathrm{F}$ & $\mathrm{M}$ & $\mathrm{F}$ & $\mathrm{M}$ & $\mathrm{F}$ & $\mathrm{M}$ & $\mathrm{F}$ \\
\hline JM1 & 1.65 & 1.67 & .95 & .98 & 1.14 & 1.24 & .30 & .22 \\
$\mathrm{JM} 2$ & 1.83 & 1.61 & .99 & .97 & .83 & 1.31 & -.61 & .38 \\
$\mathrm{JM} 3$ & 1.98 & 1.64 & 1.09 & .96 & .63 & 1.19 & -1.03 & .37 \\
$\mathrm{JM} 4$ & 1.99 & 1.92 & 1.08 & 1.11 & .64 & .77 & -.99 & -.90 \\
$\mathrm{DR} 1$ & 1.93 & 1.70 & 1.02 & 1.04 & .78 & 1.17 & -.89 & .20 \\
$\mathrm{DR} 2$ & 1.75 & 1.64 & .95 & .95 & 1.05 & 1.29 & .14 & .78 \\
$\mathrm{DR} 3$ & 1.79 & 1.75 & .88 & 1.05 & .95 & 1.04 & -.33 & -.40 \\
$\mathrm{AC} 1$ & 1.56 & 1.58 & .76 & .92 & 1.24 & 1.13 & .72 & .84 \\
$\mathrm{AC} 2$ & 1.72 & 1.47 & 1.03 & .80 & 1.16 & 1.48 & -.16 & 1.35 \\
$\mathrm{AC} 3$ & 1.63 & 1.46 & .95 & .88 & 1.18 & 1.64 & .41 & 1.63 \\
AC4 & 1.75 & 1.66 & .96 & .95 & 1.05 & 1.23 & .10 & .30 \\
\hline
\end{tabular}

Nota. $\mathrm{M}=$ masculino; $\mathrm{F}=$ femenino; $\mathrm{JM}=$ justificación moral; $\mathrm{DR}=$ difusión de la responsabilidad; $\mathrm{AC}=$ atribución de la culpa.

\section{Análisis Factorial Confirmatorio (AFC)}

Se encontraron relaciones significativas en ambos modelos entre los indicadores observables y las variables latentes. En el modelo multidimensional, las correlaciones entre los factores resultaron significativas, lo que sugiere que miden una dimensión latente común. Sin embargo, de la magnitud de estas correlaciones se infiere que miden aspectos diferentes al del constructo latente (véase Figura 2).

La diferencia en los valores de Chi-cuadrada entre ambos modelos $\left(\Delta X^{2}(3)=16.21, p=.001\right)$ resultó estadísticamente significativa, mientras que el valor de los índices de ajuste teóricos (AIC y BIC) fue menor en el modelo multidimensional, lo que sugiere un mejor ajuste a los datos en este modelo (Kline, 2016; Wang \& Wang, 2012).

\section{Invarianza de medida por sexo}

Para el análisis de la invariancia en la medición en ambos sexos se compararon cuatro modelos anidados: (a) $M_{1}$, sin restricciones (invariancia configuracional); (b) $M_{2}$, con restricciones referidas a la igualdad de pesos factoriales (invariancia métrica); (c) $M_{3}$, con restricciones relativas a las cargas factoriales e interceptos (invarianza escalar); y (d) $M_{4}$, con restricciones en lo relativo a la equivalencia entre cargas factoriales, interceptos, varianzas y covarianzas residuales (invarianza estricta).

Los valores de la Chi-cuadrada $\left(\chi^{2}=123.71, g l=80\right.$, $p=.001) \mathrm{y}$ de los índices de ajuste (CFI $=.96$; AGFI $=$ .91 ; RMSEA $=.03$, IC $90=[.02-.03])$ permiten aceptar la hipótesis de invariancia configuracional $\left(\mathrm{M}_{1}\right)$; y, con respecto a las invariancias métrica $\left(\mathrm{M}_{2}\right)$, escalar $\left(\mathrm{M}_{3}\right) \mathrm{y}$
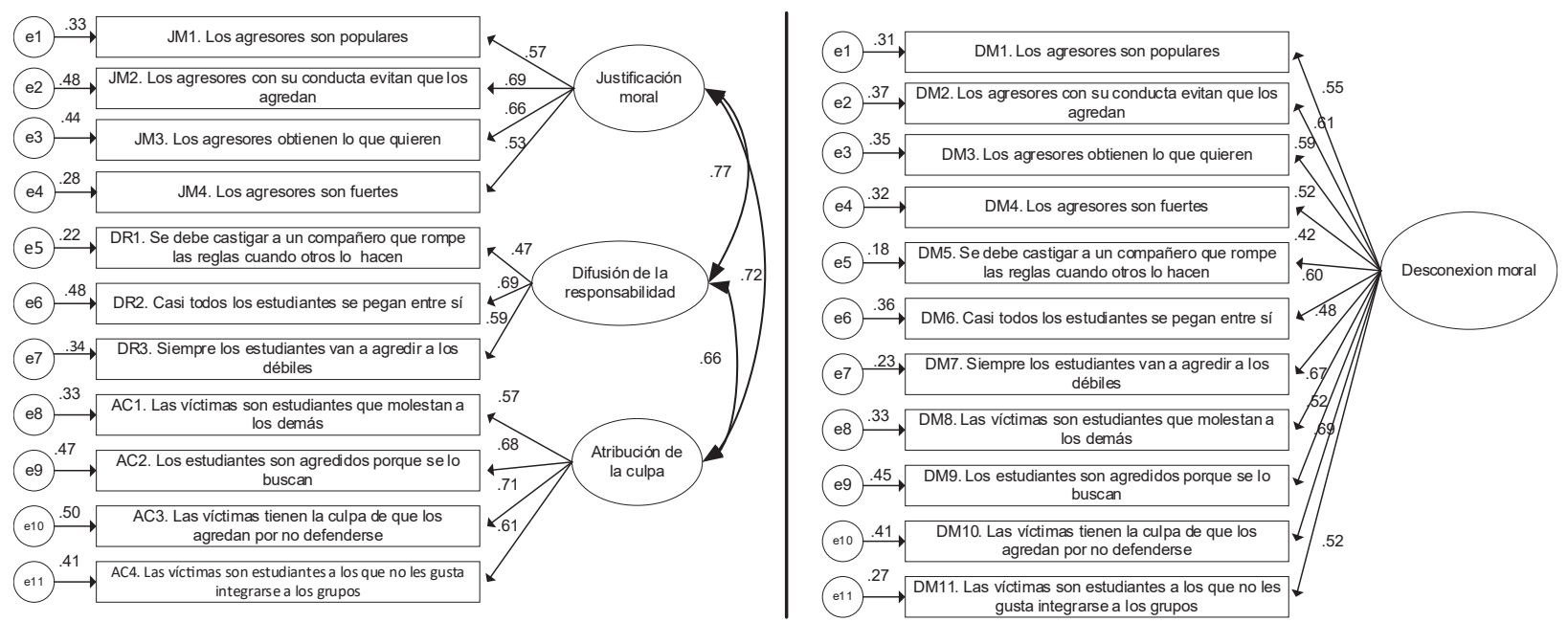

Figura 2. Resultados del análisis factorial confirmatorio de los modelos unidimensional (derecha) y tridimensional (izquierda) de medición de la DMAE.

Tabla 2.

Índices de ajuste del modelo unidimensional y tridimensional de medición de la DMAE

\begin{tabular}{lccccccccc}
\hline Modelos & $X 2$ & $g l$ & $p$ & SMRM & AGFI & CFI & RMSEA & AIC & BIC \\
\hline Modelo de un factor & 69.34 & 37 & .001 & .03 & .95 & .97 & .04 & 128.38 & 246.97 \\
& & & & & & & [.03-.06] & 128.38 & \\
Modelo de tres factores & 53.13 & 40 & .080 & .03 & .95 & .98 & $\begin{array}{c}.04 \\
{[.02-.05]}\end{array}$ & 122.23 & 228.54 \\
\hline
\end{tabular}


Tabla 3.

Resultados de invarianza por sexo de la DMAE

\begin{tabular}{ccccccc}
\hline Modelos de Invarianza & $\chi 2$ & $g l$ & $\Delta \chi 2$ & $\Delta g l$ & $p$ & $\Delta$ CFI \\
\hline M1 Configuracional & 123.71 & 80 & & & & \\
M2 Pesos factoriales (Débil) & 133.67 & 88 & 9.96 & 8 & .001 & $<.000$ \\
M3 Interceptos (Fuerte) & 139.41 & 94 & 15.7 & 14 & .002 & $<.000$ \\
M4 Residuales (Estricta) & 168.00 & 106 & 44.29 & 26 & .000 & $<.000$ \\
\hline
\end{tabular}

estricta $\left(\mathrm{M}_{4}\right)$, las probabilidades asociadas a las diferencias entre los modelos anidados $\left(\Delta \chi_{\mathrm{M} 2-\mathrm{M} 1}^{2}=9.96, \mathrm{gl}=8, \mathrm{p}<\right.$ $.267 ; \Delta \chi_{\mathrm{M} 3-\mathrm{M} 1}^{2}=15.7, g l=14, p=.332 ; \Delta \chi_{\mathrm{M} 4-\mathrm{M} 1}^{2}=44.29$, $g l=26, p=.014)$ sugieren que el modelo de medición es equivalente entre ambos sexos. Esto se confirma por las $\Delta \mathrm{CFI}$ entre los modelos anidados, ya que todas son menores a .001 (véase Tabla 3).

\section{Validez concurrente}

Para indagar sobre la validez concurrente de la DMAE se analizaron, mediante una prueba de regresión múltiple, los efectos de los mecanismos de desconexión moral en las emociones morales de empatía-compasión. De los resultados, se infiere que los mecanismos de desconexión moral (justificación moral, difusión de la responsabilidad y atribución de la culpa) se relacionan de forma negativa con las emociones morales de empatía-compasión (véase Figura 3).

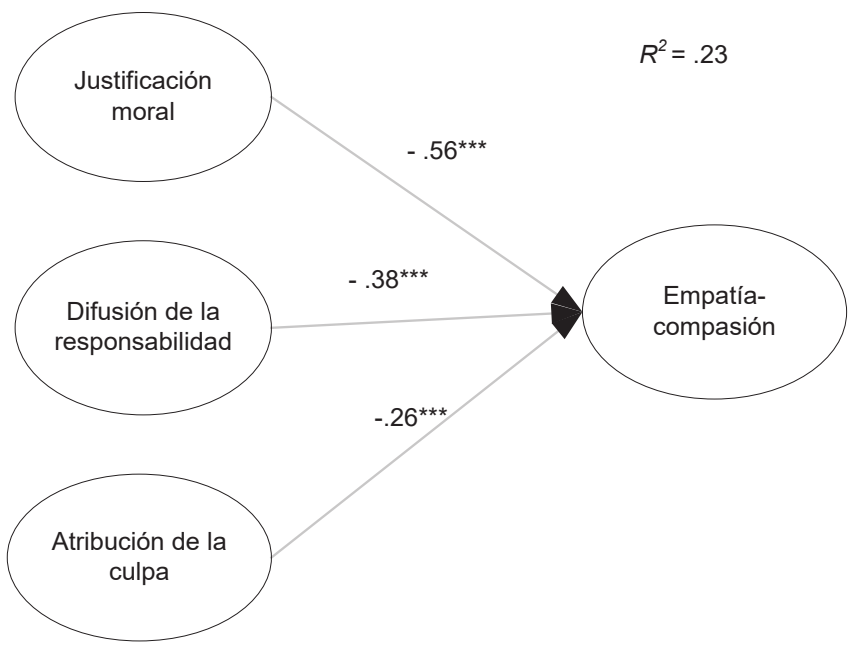

Figura 3. Resultados del modelo de regresión de las relaciones entre los mecanismos de desconexión moral y las emociones morales de empatía-compasión.

Fiabilidad de la DMAE

La fiabilidad de la DMAE se calculó mediante el coeficiente omega de McDonald $(\Omega)$ y la varianza media extractada (VME). En la Tabla 4 se puede observar que los valores de ambos estadísticos son aceptables (Dunn, Baguley \& Brunsden, 2014).

Tabla 4.

Resultados de fiabilidad de la DMAE

\begin{tabular}{cll}
\hline Mecanismos de desconexión moral & $\Omega$ & VME \\
\hline Justificación moral & .74 & $58 \%$ \\
Difusión de la responsabilidad & .70 & $52 \%$ \\
Atribución de la culpa & .76 & $61 \%$ \\
\hline
\end{tabular}

\section{Discusión}

El presente estudio se analizó las evidencias de validez (estructura interna y concurrente), invarianza de medida por sexo y fiabilidad de una escala para medir la desconexión moral en situaciones de acoso (DMAE) en niños mexicanos, y los resultados muestran que la DMAE presenta propiedades psicométricas adecuadas para la medición del constructo. Específicamente, los hallazgos proveen apoyo al modelo multidimensional de medición, la invarianza de medida para ambos sexos, la validez concurrente y la fiabilidad de los puntajes de la DMAE.

Los resultados descriptivos muestran la presencia de mecanismos de desconexión moral en niños mexicanos; no obstante, la frecuencia de utilización de estos mecanismos fue baja, tal como se espera en muestras no clínicas. Además, de forma consistente con lo reportado en la literatura, se evidencia mayor prevalencia de mecanismos de desconexión moral en los niños que en las niñas (CarreraFernández, Cid-Fernández, Almeida, González-Fernández \& Lameiras-Fernández, 2018; De Caroli \& Sagone, 2014; Malti et al., 2009).

Por otra parte, con respecto a la escala, se aprecia que el modelo de medición multidimensional se ajusta mejor a los datos que el modelo unidimensional, lo cual sugiere que la desconexión moral implica la utilización de mecanismos que, aunque relacionados, miden aspectos diferentes del constructo (Bandura et al., 1996; Newton et al., 2016; Paciello et al., 2008). Teóricamente, esto implica que es necesario analizar los factores asociados y los efectos en la conducta de cada uno de estos mecanismos, y, en el aspecto 
114

práctico, muestra la necesidad de diseñar intervenciones específicas dirigidas a desestimular el uso de las diversas formas de desconexión moral.

Adicionalmente, el valor de la escala se fortalece por su evidencia de validez concurrente, la cual se manifiesta en la relación negativa encontrada entre los mecanismos de desconexión moral y las emociones morales de empatíacompasión. Estos resultados son similares a lo reportado en la literatura acerca de los efectos negativos de la desconexión moral en las emociones morales, y, de forma particular, en la empatía y la compasión (Barriga et al., 2009; Bussey, Quinn \& Dobson, 2015; Perren \& Gutzwiller-Helfenfinger, 2012; Thornberg et al., 2015). En este sentido, es de señalar que la justificación moral presenta la relación negativa más fuerte con las emociones morales, y esto sugiere que la evaluación de la conducta agresiva como socialmente conveniente es particularmente nociva para el desarrollo moral en la infancia (Eisenberg, 2000; Menesini et al. 2003), ya que inhibe el desarrollo de la sensibilidad moral -cuyo papel es ejercer un importante efecto regulador en la conducta social- (Gasser, Gutzwiller-Helfenfinger, Latzko \& Malti, 2013; Thornberg et al., 2015).

Por otra parte, la DMAE muestra invariancia de medida en ambos sexos, lo cual resulta valioso en el estudio de la desconexión moral y atiende a una debilidad de las escalas reportadas. Este hallazgo implica que la DMAE no presenta sesgos de medición del constructo asociadas al sexo del estudiante, y que permite comparar de forma adecuada la expresión de este rasgo en niños y niñas.

El presente estudio tiene implicaciones tanto prácticas como teóricas, ya que, desde el punto de vista práctico, aporta un instrumento breve en lo que respecta al número de reactivos, además de una adecuada validez y fiabilidad para la medición de la desconexión moral en niños de educación primaria en México. Desde la perspectiva teórica contribuye a la delimitación del constructo al mostrar el valor de la medida multidimensional del mismo y permitir una comparación no sesgada de la expresión del constructo en estudiantes de ambos sexos.

Sin embargo, si bien este estudio constituye una contribución a la medición de la desconexión moral en niños, también presenta algunas limitaciones, como lo son: en primer lugar, que la DMAE es una escala de autorreporte, lo cual implica la necesidad de incluir en próximos estudios un análisis de deseabilidad social de las respuestas (NavarroGonzález, Lorenzo-Seva \& Vigil-Colet, 2016); en segundo lugar, que en la escala no se incluyen los mecanismos de desconexión moral conocidos como lenguaje eufemístico, comparaciones ventajosas, desplazamiento de la responsabilidad, distorsión de las consecuencias y deshumanización, lo cual implica la importancia de explorar otros modelos teóricos de medición de la desconexión moral; $\mathrm{y}$, en tercer lugar, que la muestra incluyó a estudiantes de escuelas primarias urbanas de una región específica, lo cual conlleva a que la generalización de los hallazgos deba ser realizada con cautela debido a la amplia diversidad étnica y cultural propia de un país como México.

Finalmente, se concluye que la DMAE, debido a sus adecuadas propiedades psicométricas, constituye una herramienta valiosa para el estudio de la desconexión moral en niños mexicanos, además de que, por su número de reactivos, resulta de fácil administración y es posible su utilización en estudios con muestras grandes. Incluso, esta escala permite a los investigadores identificar tres mecanismos de desconexión moral (justificación moral, difusión de la responsabilidad y atribución de la culpa), lo cual resulta de gran importancia práctica, ya que existe amplia evidencia de los efectos negativos de la desconexión moral en las emociones morales (Barriga et al., 2009; Thornberg et al., 2015).

\section{Referencias}

Almeida, A., Correia, L., \& Marinho, S. (2010). Moral disengagement, normative beliefs or peer group, and attitudes regarding roles in bullying. Journal of School Violence, 9, 23-36. doi: http://dx.doi.org/10.1080/15388220903185639

Aly, A., Taylor, E., \& Karnovsky, S. (2014). Moral disengagement and building resilience to violent extremism: An education intervention. Studies in Conflict and Terrorism, 37, 369385. doi: http://dx.doi.org/10.1080/1057610X.2014.879379

Arseneault, L., Walsh, E., Trzesniewski, K., Newcombe, R., Caspi, A., \& Moffitt, T. E. (2006). Bullying victimization uniquely contributes to adjustment problems in young children: A nationally representative cohort study. Pediatrics, 118, 130-138. doi: http://dx.doi.org/10.1542/peds.2005-2388

Bandura, A. (1986). Social foundations and action: A social cognitive theory. Englewood Cliffs, NJ: Prentice-Hall.

Bandura, A. (1990). Selective activation and disengagement of moral control. Journal of Social Issues, 46, 27-46. doi: http:// dx.doi.org/10.1111/j.1540-4560.1990.tb00270.x

Bandura A. (1999). Moral disengagement in the perpetuation of inhumanities. Personality and Social Psychology Review, 3, 193-209. doi: http://dx.doi.org/10.1207/s15327957ps pr0303_3

Bandura, A. (2002). Selective moral disengagement in the exercise of moral agency. Journal of Moral Education, 31, 101119. doi: http://dx.doi.org/10.1080/0305724022014322

Bandura, A., Barbaranelli, C., Caprara, G. V., \& Pastorelli, C. (1996). Mechanisms of moral disengagement in the exercise of moral agency. Journal of Personality and Social Psychology, 71, 364-374. doi: http://dx.doi.org/10.1037/00223514.71.2.364 
Bandura, A., Underwood, B., \& Fromson, M. E. (1975). Disinhibition of aggression through diffusion of responsibility and dehumanization of victims. Journal of Research in Personality, 9, 253-269. doi: http://dx.doi.org/10.1016/00926566(75)90001-X

Barriga, A. Q., Sullivan-Cosetti, M., \& Gibbs, J. C. (2009). Moral cognitive correlates of empathy in juvenile delinquents. Criminal Behaviour and Mental Health, 19, 253-264. doi: http://dx.doi.org/10.1002/cbm.740

Blunch, N. (2013). Introduction to structural equation modeling using IBM SPSS Statistics and Amos. Londres, UK: SAGE.

Boardley, I., \& Kavusannu, M. (2007). Development and validation of the Moral Disengagement in Sport Scale. Journal of Sport and Exercise Psychology, 29(5), 608-628. doi: http:// dx.doi.org/10.1123/jsep.29.5.608

Bussey, K., Quinn, C., \& Dobson, J. (2015). The moderating role of empathic concern and perspective taking on the relationship between moral disengagement and aggression. MerrillPalmer Quarterly: Journal of Developmental Psychology, 61, 10-29. doi: http://dx.doi.org/10.1353/mpq.2015.0004

Byrne, B. (2010). Structural equation modeling with AMOS $\left(2^{\text {nd }}\right.$ ed. $)$. New York, NY: Routledge Taylor \& Francis Group.

Camodeca, M., \& Taraschi, E. (2015). Like father, like son? The link between parents' moral disengagement and children's externalizing behaviors. Merril-Palmer Quarterly, 61, 173-191. doi: http://dx.doi.org/10.13110/mrrpalmquar1982.61.1.0773

Campaert, K., Nocentini, A., \& Menesini, E. (2017). The efficacy of teachers' responses to incidents of bullying and victimization: The mediational role of moral disengagement for bullying. Aggressive Behavior, 43, 483-492. doi: http://dx.doi. org/10.1002/ab.21706

Çapan, B. E., \& Bakioglu, F. (2016). Adaptation of Collective Moral Disengagement Scale into Turkish culture for adolescents. Universal Journal of Educational Research, 4, 14521457. doi: http://dx.doi.org/10.13189/ujer.2016.040624

Caravita, S. L., Sijtsema, J. J., Rambaran, J. A., \& Gini, G. (2014). Peer influences on moral disengagement in late childhood and early adolescence. Journal of Youth Adolescence, 43, 193-207. doi: http://dx.doi.org/10.1007/s10964-013-9953-1

Carrera-Fernández, M. V., Cid-Fernández, X. M., Almeida, A., González-Fernández, A., \& Lameiras-Fernández, M. (2018). Attitudes toward cultural diversity in Spanish and Portuguese adolescent of secondary education: The influence of heteronormativity and moral disengagement in school bullying. Revista de Psicodidáctica, 23, 17-25. doi: http://dx.doi. org/10.1016/j.psicod.2017.07.004

Craig, W., Harel-Fisch, Y., Fogel-Grinvald, H., Dostaler, S., Hetland, J., Simons-Morton, B., \& Molcho, M. (2009). A cross-national profile of bullying and victimization among adolescent in 40 countries. International Journal of Public Health, 54, 216-224. doi: http://dx.doi.org/10.1007/s00038-0 09-5413-9
De Caroli, M., \& Sagone, E. (2014). Belief in a just world, prosocial behavior, and moral disengagement in adolescence. Procedia-Social and Behavioral Sciences, 116, 596-600. doi: http://dx.doi.org/10.1016/j.sbspro.2014.01.263

De Graaff, M. C., Schut, M., Verweij, D. E., Vermetten, E., \& Giebels, E. (2016). Emotional reactions and moral judgment: The effects of morally challenging interactions in military operations. Ethics \& Behavior, 26, 14-31. doi: http://dx.doi. org/10.1080/10508422.2014.975815

Dunn, T., Baguley, T., \& Brunsden, V. (2014). From Alfa to Omega: A practical solution to the pervasive problem of internal consistency estimation. British Journal of Psychology, 104, 399-412. doi: http://dx.doi.org/10.1111/bjop.2046

Echavarría, C. V., \& Vasco, E. (2006). Justificaciones morales de lo bueno y lo malo en un grupo de niñas y niños provenientes de contextos violentos y no violentos de una ciudad Andina de Colombia. Acta Colombiana de Psicología, 9(1), 51-62. Recuperado de http://www.redalyc.org/ pdf/798/79890105.pdf

Eisenberg, N. (2000). Emotion, regulation, and moral development. Annual Review of Psychology, 51, 665. doi: http:// dx.doi.org/10.1146/annurev.psych.51.1.665

Eljach, S. (2011). Violencia escolar en América Latina y el Caribe: Superficie y fondo. Panamá, República de Panamá: UNICEF.

Espegale, D. L., Hong, J. S., Rao, M. A., \& Low, S. (2013). Associations between peer victimization and academic performance. Theory into Practice, 52, 233-240. doi: http://dx.doi. org $/ 10.1080 / 00405841.829724$

Feldman, M. A., Ojanen, T., Gesten, E. L., Smith-Schrandt, H., Brannick, M., Wienke, C. M., \& Alexander, L. (2014). The effects of middle school bullying and victimization on adjustment through high school: growth modeling of achievement. School attendance, and disciplinary trajectories. Psychology in the School, 51, 1046-1062. doi: http://dx.doi.org/10.1002/ pits.21799

Garaigordobil, M., \& Machimbarrena, J. M|. (2017). Stress, competence, and parental educational styles in victims and aggressors of bullying and cyberbullying. Psicothema, 29, 335-340. doi: http://dx.doi.org/10.7334/psicothema2016.258

Gasser, L., Gutzwiller-Helfenfinger, E., Latzko, B., \& Malti, T. (2013). Moral emotion attributions and moral motivation. En K. Heinrich, F. Oser, \& T. Lovat (Eds.), Handbook of moral motivation (pp. 307-322). Rotterdam: Sense Publishers.

Gibbs, J. (2014). Moral development \& reality ( $3^{\text {rd }}$ ed.). USA: Oxford University Press.

Gini, G., Pozzoli, T., \& Bussey, K. (2015). The role of individual and collective moral disengagement in peers aggression and bystanding: a multilevel analysis. Journal of Abnormal Child Psychology, 43, 441-452. doi: http://dx.doi.org/10.1007/ s10802-014 -9920-7

Gini, G., Pozzoli, T., \& Hymel, S. (2014). Moral disengagement among children and youth: A meta-analytic review of links 
116

to aggressive behavior. Aggressive Behavior, 40, 56-68. doi: http://dx.doi.org/10.1002/ab.21502

Graham, S. (2016). Victims of bullying in schools. Theory into Practice, 55, 136-144. doi: http://dx.doi.org/10.1080/00405 841.2016.1148988

Greenhalgh, E. M., Watt, S. E., \& Schutte, N. S. (2015). Mechanisms of moral disengagement in the endorsement of asylum seeker policies in Australia. Ethics \& Behavior, 25, 482-499. doi: http://dx.doi.org/10.1080/10508422.2014.951720

Higuita-Gutiérrez, L. F., \& Cardona-Arias, A. (2017). Variables of the family, school, and social environment context that determine bullying in adolescents in Medellin, Colombia, 2014. Journal of School Violence, 16, 1-19. doi: http://dx.doi. org/10.1080/1 53882202015.1112807

Houwing, F., \& Bussey, K. (2017). Moral disengagement and the propensity to endorse physical punishment practices. Journal of Child \& Family Studies, 26, 1206-1218. doi: http://dx.doi. org/10.1007/s10826-016-0643-y

Hymel, S., \& Bonanno, R. A. (2014). Moral disengagement processes in bullying. Theory into Practice, 53, 278-285. doi: http://dx.doi.org/10.1080/00405 841.2014.94 7219

Instituto Nacional de Estadística y Geografía (2015). Encuesta de cohesión social para la prevención de la violencia y delincuencia. Ciudad de México, México: INEGI.

Kim, M. J., Catalano, R. F., Haggerty, K. P., \& Abbott, R. D. (2011). Bullying at elementary school and problem behavior in young adulthood: A study of bullying, violence and substance abuse from 11 to age 21. Criminal Behaviour and Mental Health, 21, 136-144. doi: http://dx.doi.org/10.1002/ cbm.804

Kline, R. B. (2016). Principles and practice of structural equation modeling ( $4^{\text {th }}$ ed.). New York, NY: The Guilford Press.

Kokkinos, C. M., Voulgaridou, I., Mandrali, M., \& Parousidou, C. (2016). Interactive links between relational aggression, theory of mind, and moral disengagement among early adolescents. Psychology in the Schools, 53, 253-269. doi: http:// dx.doi.org/10.1 002/pits.21902

Levasseur, C., Desbiens, N., \& Bowen, F. (2017). Moral reasoning about school bullying in involved adolescent. Journal of Moral Education, 46, 158-176. doi: http://dx.doi. org/10.1080/030 57240.2016.1268113

Malti, T., Gasser, L., \& Buchmann, M. (2009). Aggressive and prosocial children's emotion attributions and moral reasoning. Aggressive Behavior, 35, 90-102. doi: http://dx.doi. org/10.1002/ab.20289

Malti, T., \& Ongley, S. F. (2014). The development of moral emotions and moral reasoning. En M. Killen, \& J. G. Smetana (Eds.), Handbook of moral development ( $2^{\text {nd }}$ ed., pp. 163183). New York, NY: Psychology Press.

Mendoza, B., Rojas, C., \& Barrera, A. (2017). Rol de participación en bullying y su relación con la ansiedad. Perfiles Educativos, XXXIX(158), 38-51.
Menesini, E., Palladino, B. E., \& Nocentini, A. (2015). Emotions of moral disengagement, class norms, and bullying in adolescence: A multilevel approach. Merrill-Palmer Quarterly: Journal of Developmental Psychology, 61, 124-143. doi: http://dx.doi.org/10.13110/merrpalmquar1982.61.1.0124

Menesini, E., Sanchez, V., Fonzi, A., Ortega, R., Costabile, A., \& Lo Feudo, G. (2003). Moral emotions and bullying: A crossnational comparison of differences between bullies, victims and outsiders. Aggressive Behavior, 29, 515-530. doi: http:// dx.doi.org/10.1002/ab.10060

Moore, C. (2015). Moral disengagement. Current Opinion in Psychology, 6, 199-204. doi: http://dx.doi.org/10.1016/j.copsyc.2015.07.018

Navarro-González, D., Lorenzo-Seva, U., \& Vigil-Colet, A. (2016). How response bias affects the factorial structure of personality self-reports. Psicothema, 29, 465-470. doi: http:// dx.doi.org/10.7334/psicothema2016.113

Newton, N. C., \& Bussey, K. (2012). The age of reason: An examination of psychosocial factors involved in delinquent behaviour. Legal \& Criminological Psychology, 17, 75-88. doi: http://dx.doi.org/10.1111/j.2044-8333.2010.02004.x

Newton, N. C., Havard, A., \& Teesson, M. (2012). The association between moral disengagement, psychological distress, resistive self-regulatory efficacy and alcohol and cannabis use among adolescents in Sydney, Australia. Addiction Research \& Theory, 20, 261-269. doi: http://dx.doi.org/10.3109 /16066359.2011.614976

Newton, N. C., Stapinski, L. A., Champion, K. E., Teesson, M., \& Bussey, K. (2016). The reliability and validity of the Australian Moral Disengagement Scale. Behaviour Change, 33, 136-149. doi: http://dx.doi.org/10.1017/bec.2016.9

Nucci, L. P. (2001). Education in the moral domain. Cambridge: Cambridge University Press.

Obermann, M. L. (2011). Moral disengagement among bystander of school bullying. Journal of School Violence, 10, 239257. doi: http://dx.doi.org/10.1080/15388220.2011. 578276

Olweus, D. (1991). Bully/victim problems among schoolchildren: Basic facts and effects of a school based intervention program. En D. Pepler \& K. Rubin (Eds.), The development and treatment of childhood aggression (pp. 411-448). Hillsdale, NJ: Erlbaum.

Paciello, M., Fida, R., Tramontano, C., Lupinetti, C., \& Caprara, G. V. (2008). Stability and change of moral disengagement and its impact on aggression and violence in late adolescence. Child Development, 79, 1288-1309. doi: http://dx.doi. org/10.1111/ j. 1467-8624.2008.01189.x

Passini, S. (2012). The delinquency-drug relationship: The influence of social reputation and moral disengagement. Addictive Behaviors, 37, 577-579. doi: http://dx.doi.org/10.1016/j.addbeh.2012.01.012

Paulino, L. R., Avilés, J. M., \& Fonseca, P. J. (2016). Bullying, un problema moral: representaciones de sí mismo y desconexiones morales. Revista de Educación (373), 9-34. doi: http:// dx.doi.org/10.4438/1988-592X-RE-2016-373-319 
Paxton, J. M., \& Greene, J. D. (2010). Moral reasoning: hints and allegations. Topics in Cognitive Science, 2, 511-527. doi: http://dx.doi.org/10.1111/j.1756-8765.2010.01096.x

Pelton, J., Gound, M., Forehand, R., \& Brody, G. (2004). The Moral Disengagement Scale: Extension with an American minority sample. Journal of Psychopathology \& Behavioral Assessment, 26, 31-39. doi: http://dx.doi.org/10.1023/ B:JOBA.000

Perren, S., \& Gutzwiller-Helfenfinger, E. (2012). Cyberbullying and traditional bullying in adolescence: Differential roles of moral disengagement, moral emotions, and moral values. European Journal of Developmental, 9, 195-209. doi: http:// dx.doi.org/10.1080/17405629.2011.643168

Perren, S., Gutzwiller-Helfenfinger, E., Malti, T., \& Hymel, S. (2012). Moral reasoning and emotion attributions of adolescent bullies, victims, and bully-victims. British Journal of Developmental Psychology, 30, 511-530. doi: http://dx.doi. org/10.1111/j. 2044 -83 5X.2011.02059.x

Pozzoli, T., Gini, G., \& Vieno, A. (2012). Individual and class moral disengagement in bullying among elementary school children. Aggressive Behavior, 38, 378-388. doi: http:// dx.doi.org/10.1002/ab.21442

Robson, C., \& Witenberg, R. T. (2013). The influence of moral disengagement, morally based self-esteem, age, and gender on traditional bullying and cyberbullying. Journal of School Violence, 12, 211-231. doi: http://dx.doi. org/10.1080/15388220.201212.762921

Romero, M. L., \& Kyriacou, C. (2016). Self-efficacy and moral disengagement in Mexican secondary school bullying bystanders. Psychology of Education Review, 40(2), 28-32.

Rubio-Garay, F., Amor, P. J., \& Carrasco, M. A. (2017). Dimensionality and psychometric properties of the Spanish version of the Mechanisms of Moral Disengagement Scale (MMDS-S). Revista de Psicopatología y Psicología Clínica, 22, 43-54. doi: http://dx.doi.org/10.5944/rppc.vol.22. num.1.2017.16014

Smetana, J. C. (2006). Social-cognitive domain theory: consistencies and variations in children's moral and social judgments. En J. C. Smetana \& M. Killen (Eds.), Handbook of moral development (pp. 119-153). New Jersey: Erlbaum.

Smetana, J. C. (2013). Moral development: The social domain theory view. En P. Zelazo (Ed.), Oxford handbook of developmental psychology: Vol 2: Self and other (pp. 832-865). New York, NY: Oxford University Press.

Smetana, J. C., Jambon, M., \& Ball, C. (2014). The social domain approach to children moral and social judgments. En M. Killen, \& J. G. Smetana (Eds.), Handbook of moral development ( $2^{\text {nd }}$ ed., pp. 23-45). New York, NY: Psychology Press.

Stevens, G., Deuling, J., \& Armenakis, A. (2012). Successful psychopaths: Are they unethical decision-makers and why? Journal of Business Ethics, 105, 139-149. doi: http://dx.doi. org/10.1007/s10551-011-0963-1
Tangney, J. P., Stuewig, J., \& Mashek, D. J. (2007). Moral emotions and moral behavior. Annual Review of Psychology, 58, 345-372. doi: http://dx.doi.org/10.1146/ annurev. psych.56.091103.070145

Thornberg, R., \& Jungert, T. (2014). School bullying and the mechanisms of moral disengagement. Aggressive Behavior, 40, 2, 99-108. doi: http://dx.doi.org/10.1002/ ab.21509

Thornberg, R., Pozzoli, T., Gini, G., \& Jungert, T. (2015). Unique and interactive effects of moral emotions and moral disengagement on bullying and defending among school children. The Elementary School Journal, 116, 322-337. doi: http:// dx.doi.org/10.1086/683985

Valdebenito, S., Ttofi, M., \& Eisner, M. (2015). Prevalence rates of drug use among school bullies and victims: A systematic review and meta-analysis of cross-sectional studies. Aggression and Violent Behavior, 23, 137-146. doi: http://dx.doi. org/10.1016/j.avb.2015.05.004

Valdés, A. A., Carlos, E. A., Wendlandt, T. R., \& Ramírez, M. (2016). Propiedades psicométricas de una escala para medir el manejo de la vergüenza en adolescents (MOSS-SAST). Acta Colombiana de Psicología, 19, 13-23. doi: http://dx.doi. org/10.14718/ACP.2016.19.1.2

Valdés, A. A., Martínez, B., \& Carlos, E. A. (2018). The role of teaching practices in the prevention of school violence among peers. Revista de Psicodidáctica, 23, 33-38. doi: http://dx.doi.org/10.1016/j.psicod.2017.05.006

Van Noorden, T. H., Haselager, G. J., Cillessen, A. H., \& Bukowski, W. M. (2014). Dehumanization in children: The link with moral disengagement in bullying and victimization. Aggressive Behavior, 40, 320-328. doi: http://dx.doi. org/10.1002/ab.215 22

Volk, A. A., Dane, A. V., \& Marini, Z. A. (2014). What is bullying? A theoretical redefinition. Developmental Review, 34, 327-343. doi: http://dx.doi.org/10.1016/j.dr.2 014.09.001

Vossen, H. G. M., Piotrowski, J. T., \& Valkenburg, P. M. (2015). Development of the Adolescent Measure of Empathy and Sympathy (AMES). Personality and Individual Differences, 74, 66-71. doi: http://dx.doi.org/10.1016/j.paid.2014.09.040

Wang, C., Ryoo, J., Swearer, S., Turner, R., \& Goldberg, T. (2017). Longitudinal relationships between bullying and moral disengagement among adolescents. Journal of Youth and Adolescence, 46, 1304-1317. doi: http://dx.doi.org/10.1007/s10 964-016-0577-0

Wang, X., Yang, L., Gao, L., Lei, L., \& Wang, C. (2017). Childhood maltreatment and Chinese adolescents' bullying and defending: The mediating role of moral disengagement. Child Abuse \& Neglect, 69, 134-144. doi: http://dx.doi. org/10.1016/ j.chiabu.2017.04.016

Wang, J., \& Wang, X. (2012). Structural equation modeling. Applications using Mplus. Chichester, UK: Wiley. 